\title{
Experimental Study on Energy Consumption and Performance of Hydroxyethyl Ethylenediamine Solution for $\mathrm{CO}_{2}$ Capture
}

\author{
Bingcheng Liu ${ }^{1}$, Ting Wang ${ }^{*}$, Xuan Yang ${ }^{1}$, Pen-Chi Chiang ${ }^{2}$ \\ ${ }^{1}$ Qingdao University of Science and Technology, Qingdao 266061, China \\ ${ }^{2}$ Carbon Cycle Research Center, National Taiwan University, Taipei City 10672, Taiwan
}

\begin{abstract}
Chemical adsorption is a mature post-combustion technology for $\mathrm{CO}_{2}$ capture from large-scale power plants. At present, the traditional monoethanola mine (MEA) absorbent has a few drawbacks, including a low absorption rate, solvent degradation, and high energy consumption, which can restrict the development of absorption technology. Therefore, the application of hydroxyethyl ethylenediamine (AEE) has attracted increasing attention in recent years. In the past, the absorbent performance test was usually measured for a single use, where changes in the performance were rarely mentioned after several absorbent cycles. In the current work, the absorption and desorption performance of AEE absorbents at different concentrations for multiple cycles are investigated. The experimental results indicate that (1) $10 \mathrm{wt} \%$ AEE has a higher absorption rate; (2) $20 \mathrm{wt} \%$ AEE has a higher absorption, desorption rate, and degree of regeneration, but has higher energy consumption. (3) When the temperature reaches about $85^{\circ} \mathrm{C}$, the desorption rate of three different concentrations reaches the highest level, and the desorption rate increases significantly with increases in the solution concentration. (4) After several cycles, the desorption rate and energy consumption of AEE solutions are always keep a great state, so the AEE solution can be used repeatedly.
\end{abstract}

Keywords: $\mathrm{CO}_{2}$ capture; Chemical absorption; Hydroxyethyl ethylenediamine (AEE); Energy consumption; Capture performances.

\section{INTRODUCTION}

Recently, global climate change caused by large amounts of greenhouse gas emissions has attracted increasing concern. $\mathrm{CO}_{2}$, as one of the most important greenhouse gases, plays a significant role in climate change (Anderson et al., 2004; Szulczewski et al., 2012; Yu et al., 2012; Lee et al., 2017; Su et al., 2017; Yang et al., 2017; Ghasemifard et al., 2019). According to the Global Carbon Project (GCP, 2016), China was the source of $29 \%$ of the total global $\mathrm{CO}_{2}$ emissions in 2015 as the largest emitter, at nearly twofold that of the secondplaced United States (15\%) (Liu et al., 2018). Therefore, it is essential to develop effective technology to mitigate $\mathrm{CO}_{2}$ emissions (Butterfield et al., 2017). $\mathrm{CO}_{2}$ capture, utilization, and storage (CCUS) is a promising technology to mitigate global warming by capturing $\mathrm{CO}_{2}$ from coal fired power plants, storing it, and reusing it with the effect of reducing about $80 \%-90 \%$ of the $\mathrm{CO}_{2}$ emissions from coal-fired power plants (Li et al., 2013; Li et al., 2016; IEA, 2017; Beidari et al., 2017; Jain et al., 2018). Currently, different $\mathrm{CO}_{2}$ capture

\footnotetext{
${ }^{*}$ Corresponding author.

E-mail address: 17864212328@163.com
}

and separation methods with low energy consumption and high capture efficiency have been widely researched (Chatterjee et al., 2018).

In recent years, the detailed $\mathrm{CO}_{2}$ capture methods include absorption, adsorption, membrane, and cryogenic methods (Lund et al., 2012; Song et al., 2012; Pan et al., 2013). Although these technologies are widely used for $\mathrm{CO}_{2}$ capture from flue gas, there are also some bottlenecks in these technologies. In the case of physical adsorption, the limitations are mainly low $\mathrm{CO}_{2}$ selectively and removal efficiency and problems with adsorbent and adsorbent regeneration capacity (Yancheshmeh et al., 2016; Bamdad et al., 2018). Membrane methods comprise a mature technology for $\mathrm{CO}_{2}$ capture from coal-fired power plants, but they also have some drawbacks: 1) the membrane structure has a high temperature requirement, and 2) the membrane material is sensitive to $\mathrm{SO}_{\mathrm{x}}, \mathrm{NO}_{\mathrm{x}}, \mathrm{H}_{2} \mathrm{~S}$, etc. (Zhang et al., 2013; Wang et al., 2017). The main restriction for cryogenic technology is higher energy consumption (Monda et al., 2012). In comparison to other capture technologies, because of its high efficiency, large capacity, and efficient removal effects, chemical absorption using aqueous alkanolamine solutions has been proposed as the most applicable technology for $\mathrm{CO}_{2}$ capture prior to 2030 (Rochelle, 2009; Yu et al., 2012).

Alkanolamine solutions used for $\mathrm{CO}_{2}$ capture have been 
widely studied and applied by scholars (Hikita et al., 1979; Azhgan et al., 2016; Xie et al., 2017; Melnikov et al., 2019). Among the alcohol-amines, there are primary amines such as monoethanolamine (MEA); the secondary amines include diethanolamine (DEA) and di-2-propanol-amine, and the tertiary amines include methyldietha-nolamine (MDEA). Specifically, aqueous MEA solutions have been extensively used because of their high reactivity, low solvent cost, easy reclamation, and the low absorption of hydrocarbons, as well as a high $\mathrm{CO}_{2}$ capture rate of approximately $90 \%$ via a strong chemical reaction (Issacs et al., 1980; Zhang et al., 2018). However, MEA absorption has several disadvantages, including a high volume occupancy, significant energy consumption, low solvent regeneration efficiency, corrosion during solvent regeneration, and the formation of components such as formaldehyde, acetic acid, hydroxyacetaldehyde, glycolic acid, and formic acid, among others, that cannot be regenerated by thermal heat, which reduces the $\mathrm{CO}_{2}$ removal efficiency and increases the operational costs (Goff et al., 2004; Lin et al., 2014; Shakerian et al., 2015; Afkhamipour et al., 2019).

Absorbent performance limits the large-scale popularization of $\mathrm{CO}_{2}$ capture of power plant flue gas. Therefore, it is necessary to find a $\mathrm{CO}_{2}$ absorbent with excellent performance to improve the of alcohol amine solutions to absorb $\mathrm{CO}_{2}$. Research activities are ongoing to find effective solvents that provide high $\mathrm{CO}_{2}$ loading, a high absorption rate, good mass transfer performance, lower corrosion rates, and low degradation tendencies (Liang et al., 2015). Kato et al. (2013) evaluated $\mathrm{CO}_{2}$ absorption through various amine-based experiments. Their results indicated that hindering of amines results in high $\mathrm{CO}_{2}$ absorption capacity, low reaction heat, and the required regeneration energy. Danielle Bonenfant et al. (2005) studied $\mathrm{CO}_{2}$ absorption/desorption rates in various aqueous amine solutions consisting of AEE, MEA, and blends of AEE and MDEA at different concentrations to compare the $\mathrm{CO}_{2}$ loading and recuperation properties of these amines. Their results suggested that the structural properties of AEE, including the presence of two amine groups, as compared to MEA could contribute to its $\mathrm{CO}_{2}$ absorption and recuperation capacities. Gai et al. (2008) selected AEE as the absorbent, and the results showed that the amine group in AEE had better $\mathrm{CO}_{2}$ absorption ability than that in MEA. However, AEE is worse than MEA in terms of desorption ability. In spite of this, at the same concentration, regenerated AEE has better $\mathrm{CO}_{2}$ absorption capacity than regenerated MEA. Therefore, the application of hydroxyethyl ethylenediamine (AEE) has attracted increasing attention in recent years. At the same time, absorbent performance tests typically measure the single-use performance of the absorbent, and the changes in performance after several cycles are rarely mentioned. In the actual selection of the alcohol amine absorbent process, not only is adsorption required, but recipients also should have good absorption and desorption properties when they are first used. At the same time, the solution should maintain good performance and stability in the process of repeated cyclic adsorption and desorption.

In this paper, the absorption and desorption performance of AEE solvents at different concentrations of $10 \mathrm{wt} \%$,
$15 \mathrm{wt} \%, 20 \mathrm{wt} \%$ were studied. At the same time, in order to get closer to industrial production, three cycles of absorption and desorption of the alcohol amine solution at the same concentration were carried out, and the difference between the performance of the reagents after cyclic absorption and desorption and the performance of the initial fresh reagents were compared so as to provide more accurate basis for the improvement of the industrial reagents.

\section{EXPERIMENTAL PROCEDURE}

\section{Experimental Mechanism and Materials}

Primary and secondary amines have the same reaction mechanism, which has been studied for a long time and is widely recognized (Jamal et al., 2006). In general, the reaction between $\mathrm{CO}_{2}$ and primary and secondary alkanolamines in aqueous solutions can be described by the zwitterion mechanism, which was originally proposed by Caplow (1968) and reintroduced by Danckwerts (1979).

The principle of $\mathrm{CO}_{2}$ absorption by organic amines is a reversible process in which weak acids react with weak bases to form salts in aqueous solution. The reaction mechanism of $\mathrm{CO}_{2}$ absorption by AEE (secondary amine) is as follows:

$$
\begin{aligned}
& 2 \mathrm{H}_{2} \mathrm{O} \leftrightarrow \mathrm{H}_{3} \mathrm{O}^{+}+\mathrm{OH}^{-} \\
& \mathrm{CO}_{2}+\mathrm{OH}^{-} \leftrightarrow \mathrm{HCO}_{3}^{-} \\
& \mathrm{CO}_{2}+\mathrm{H}_{2} \mathrm{O} \leftrightarrow \mathrm{HCO}_{3}^{-}+\mathrm{H}^{+} \\
& \mathrm{CO}_{2}+2 \mathrm{R}_{4} \mathrm{R}_{5} \mathrm{NH} \Leftrightarrow \mathrm{R}_{4} \mathrm{R}_{5} \mathrm{NH}_{2}{ }^{-}+\mathrm{R}_{4} \mathrm{R}_{5} \mathrm{NCOO}^{-}
\end{aligned}
$$

The above Eqs. (1)-(4) are reversible reactions. When the ambient temperature rises, the carbamate and $\mathrm{HCO}_{3}{ }^{-}$in the solution decompose by heating. The reaction proceeds in the reverse direction, and $\mathrm{CO}_{2}$ gas is released from the solution, which is the mechanism of the regeneration reaction.

\section{Absorption Experiment}

The flowchart of the absorption experiment is shown in Fig. 1. The reagents and gas of experiment is shown in Table 1. Experiments were carried out in hybrid gas obtained by mixing the content of cylinders 1 and 2 to simulate flue gas ( 85 vol. $\%$ and 15 vol. $\%$ of $\mathrm{N}_{2}$ and $\mathrm{CO}_{2}$, respectively). The mass flow controllers ( 5 and 6 ) accurately controlled the flow of mixed gas that then entered the gas mixing buffer cylinder (7). After full mixed in the gas mixing cylinder, the gas was absorbed in a double-layer glass reactor. The hightemperature circulator (16) provided a circulating water bath with adjustable temperature. The temperature could be accurately measured by the thermocouples (11 and 12) with an accuracy of $0.1^{\circ} \mathrm{C}$. The unabsorbed gas was discharged through the pipeline above the reactor and then entered the saturated calcium hydroxide solution for tail gas recovery.

\section{Desorption Experimental Procedure}

Fig. 2 is the flow chart of the desorption experiment. First, the high-temperature circulator was opened (11) after the 
synthetic heat-conducting silicone oil was heated to the predetermined temperature. Then, the valve of the hightemperature circulator was opened (10), the intelligent turbine flowmeter (8) was observed, and the cycle heating oil valve (10) was adjusted to the predetermined flow rate. After the readings of the PT100 thermocouple (4 and 6) were stable
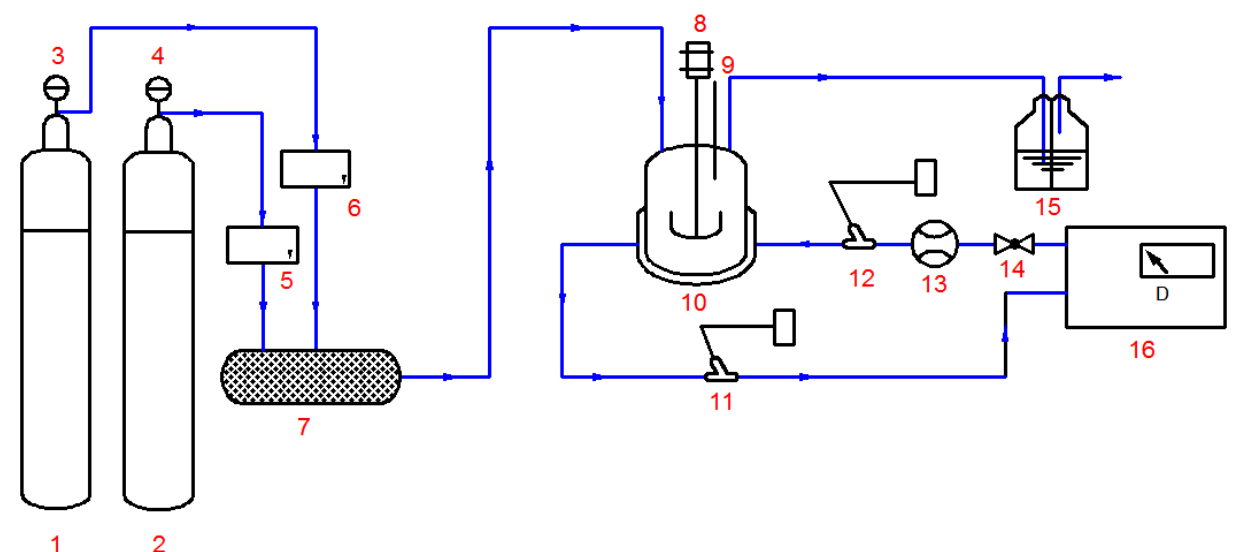

1. $\mathrm{CO}_{2}$ cylinder; 2. $\mathrm{N}_{2}$ cylinder; 3, 4. Gas decompression valve; 5, 6. Flow controller; 7. Gas mixing buffer tank; 8. Magnetic agitator; 9. Thermometer; 10. Double glass reactor; 11, 12. PT100 thermocouple; 13. Intelligent turbine flowmeter; 14. Ball valve; 15 . New saturated calcium hydroxide solution; 16 . High-temperature circulator

Fig. 1. Flowsheet of the AEE absorption experiment.

Table 1. Reagents and gas of experiment.

\begin{tabular}{ll}
\hline Main materials & Specifications \\
\hline Hydroxyethyl ethylenediamine (AEE) & Analytical reagent (AR) \\
Sulfuric acid $\left(\mathrm{H}_{2} \mathrm{SO}_{4}\right)$ & Analytical reagent (AR) \\
Methyl orange & Analytical reagent (AR) \\
Nitrogen $\left(\mathrm{N}_{2}\right)$ & $\geq 99 \%$ \\
Carbon dioxide $\left(\mathrm{CO}_{2}\right)$ & $\geq 99 \%$ \\
\hline
\end{tabular}

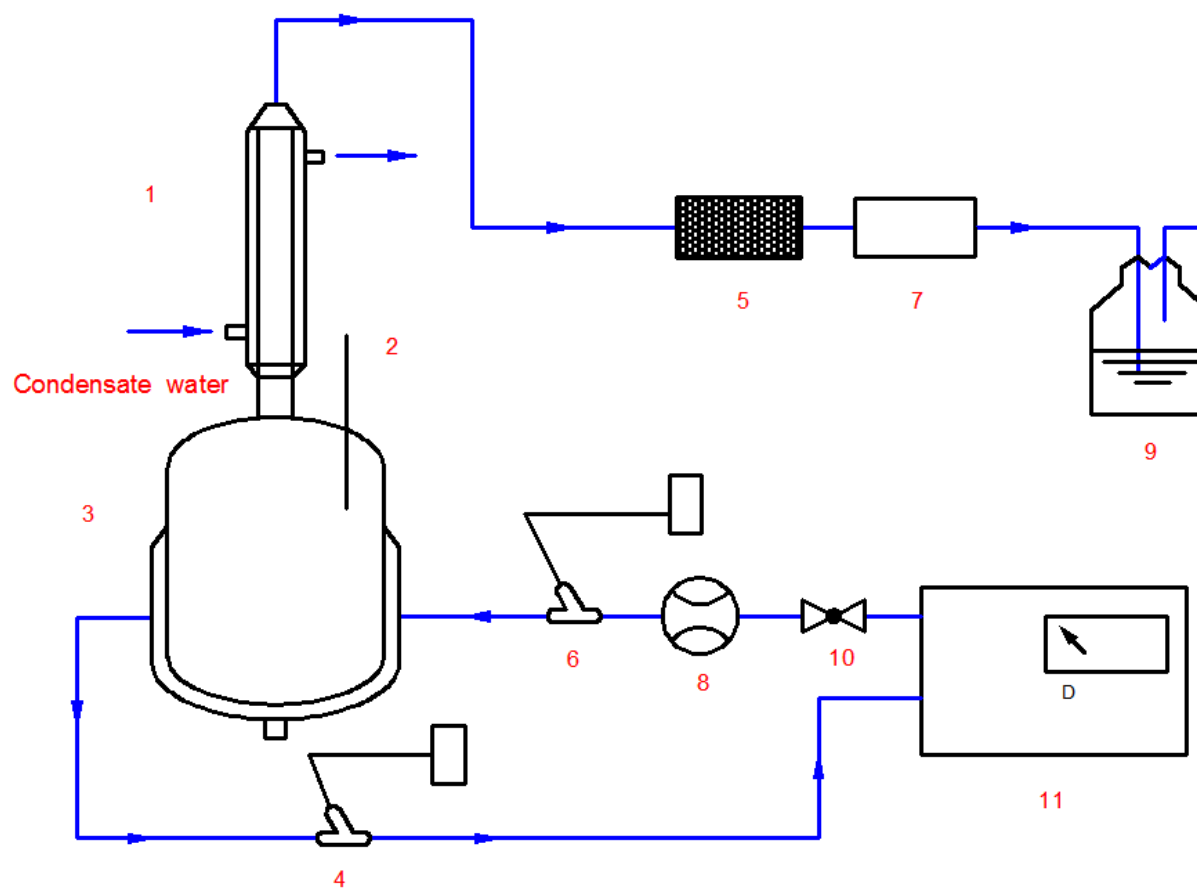

1. Condensation tube; 2. Thermometer; 3. Double glass reactor; 4, 6. PT100 thermocouple; 5. Dryer; 7. Gas flow meter; 8. Intelligent turbine flowmeter; 9. New saturated calcium hydroxide solution; 10. Ball valve; 11. High-temperature circulator

Fig. 2. Flowsheet of the AEE desorption experiment. 
(i.e., the temperatures of the inlet and outlet of the circulating heating oil in the double glass reactor were stable), the rich liquid obtained after the absorption reaction was rapidly injected into the reactor, and the sealing plug was capped for the desorption experiment. The gas released from desorption entered the dryer (5) through the serpentine condensation tube (1), entered the wet gas flow meter (7) for measurement, and finally entered the saturated calcium hydroxide solution for tail gas absorption. A standard mercury thermometer (accurate to $0.1^{\circ} \mathrm{C}$ ) was used to measure the solution temperature in real time during the experiment.

\section{Measurement of the Experimental Parameters \\ Determination of $\mathrm{CO}_{2}$ Content in the Alcohol Amine Solution}

$\mathrm{CO}_{2}$ in the rich liquid is in the form of either carbonate or bicarbonate. When it interacts with a strong acid, it is released, and the volume of the $\mathrm{CO}_{2}$ can be measured. Then, the volume will be corrected according to the ambient temperature and atmospheric pressure at that time, so that the total amount of $\mathrm{CO}_{2}$ can be calculated.

$$
\begin{aligned}
& \mathrm{CO}_{3}{ }^{2-}+2 \mathrm{H}^{+}=\mathrm{H}_{2} \mathrm{O}+\mathrm{CO}_{2} \\
& \mathrm{HCO}_{3}{ }^{-}+\mathrm{H}^{+}=\mathrm{H}_{2} \mathrm{O}+\mathrm{CO}_{2} \\
& C=\frac{V}{V_{0}} \times \frac{P-P_{1}}{P_{0}} \times \frac{T}{T+t^{\prime}}
\end{aligned}
$$

where $C$ is the volume concentration of $\mathrm{CO}_{2}$ in the liquid phase. $V$ and $V_{0}$ are the sampling quantities of the test solution and the quantity after the experiment, respectively. $T$ is the absolute temperature of the ideal gas. $P_{0}$ is the standard atmosphere, and $P$ and $P_{1}$ are atmospheric pressure at the time of measurement and the saturated vapor pressure at $t^{\circ} \mathrm{C}$, respectively.

\section{Absorption Rate}

The absorption rate $(N)$ is the amount of $\mathrm{CO}_{2}$ absorbed by the alcohol amine solution per unit time. In the experiment, $\mathrm{CO}_{2}$ is regarded as an ideal gas, where the absorption rate can be calculated as follows:

$$
N=\frac{P\left(\frac{Q_{\text {vout }}}{1-y_{\text {out }}}\right) y_{\text {out }}-Q_{\text {Vin }}}{R T},
$$

where $p$ is the pressure of $\mathrm{CO}_{2}$, in units of one atmosphere. $Q_{v o u t}$ and $Q_{\text {vin }}$ are the volume flow of the inlet and outlet $\mathrm{CO}_{2}$, respectively. $R$ is a constant; $T$ is the absolute temperature $(293 \mathrm{~K})$, and $y_{\text {out }}$ is the volume fraction of the $\mathrm{CO}_{2}$ in the export mixture gas.

\section{Determination of Desorption Energy Consumption}

The desorption energy consumption of rich liquid is calculated based on the heat loss of circulating heating oil in the desorption process. In the experiment, the desorption reactor should be well insulated, and the temperature difference between the heating oil inlet and outlet should be kept within $0.2^{\circ} \mathrm{C}$ without adding the solution into the reactor. This ensures that the heat loss of the oil is minimal. The loss is eliminated by measuring the temperature difference between the inlet and outlet oil of the reactor before the desorption reaction. The data that must be measured include: the oil temperature at the inlet and outlet, the circulating heating oil flow rate, the $\mathrm{CO}_{2}$ content in the solution, and the desorption energy consumption. The calculation formula for desorption energy consumption is as follows:

(1) Total desorption energy consumption

$E=C \rho Q_{\nu}\left(T_{2}-T_{1}\right)$,

where $\mathrm{E}$ is total desorption energy consumption; $C$ is the specific heat of the heating oil' $\rho$ is density of the heating oil; $Q_{v}$ is the volume flow of the heating oil, and $T_{1}$ and $T_{2}$ are inlet and outlet temperature of the heating oil, respectively.

(2) Unit desorption energy consumption

$E^{\prime}=\Delta E /\left(10^{-6} \times \rho^{\prime} V_{C O 2}\right)$,

where $E^{\prime}$ is the unit desorption energy consumption; $\Delta E$ is the desorption energy consumption for each time period; $V_{\mathrm{CO} 2}$ is the volume of $\mathrm{CO}_{2}$ extracted during each time period, and $\rho$ is the density of $\mathrm{CO}_{2}\left(1.977 \mathrm{~g} \mathrm{~L}^{-1}\right)$.

\section{Degree of Regeneration for the Solution}

The degree of regeneration reflects the repeated absorption and desorption effects of the absorbent in the recycling process. It is an important parameter by which to measure the regeneration effect of the absorbent. The degree of regeneration is defined as the ratio of the amount of $\mathrm{CO}_{2}$ extracted by desorption in the process of solution desorption to the amount of $\mathrm{CO}_{2}$ in the rich solution, which can be calculated as follows:

$\delta=\frac{(M-C)}{M} \times 100 \%$

where $M$ is $\mathrm{CO}_{2}$ content in the rich liquid; $C$ is the content of $\mathrm{CO}_{2}$ in the solution, and $\delta$ is the degree of regeneration.

\section{RESULTS AND DISCUSSION}

\section{Experiment on $\mathrm{CO}_{2}$ Absorption Using AEE Solutions}

In order to objectively evaluate the $\mathrm{CO}_{2}$ absorption performance of AEE solutions at different concentrations at room temperature and pressure, AEE solutions with mass fractions of $10 \mathrm{wt} \%, 15 \mathrm{wt} \%, 20 \mathrm{wt} \%$ were selected for the $\mathrm{CO}_{2}$ absorption experiments. The results are shown in Figs. 3 to 5 .

Fig. 3 shows that the absorption rate of $\mathrm{CO}_{2}$ by the AEE solution is related to the mass fraction of AEE in the same reaction time, where the trend of the three curves is basically the same. The low mass fraction $(10 \mathrm{wt} \%)$ AEE solution has a higher initial absorption rate. As the absorption reaction 
proceeds, the absorption rate of the low mass fraction (10 wt $\%$ ) AEE solution decreases more significantly. When the reaction lasts for about 30 minutes, the absorption rate of the low mass fraction $(10 \mathrm{wt} \%)$ AEE solution is the lowest. The initial absorption rate of the solution was related to two factors: the concentration of the solution, and the degree of diffusion of the $\mathrm{CO}_{2}$ gas in the liquid phase. The lower absorption rate of the high concentration solution was determined by the viscosity of the solution. The higher viscosity of the high concentration solution affected the diffusion of $\mathrm{CO}_{2}$ in the solution. In this study, the high mass fraction (20 wt \%) AEE solution had a relatively high $\mathrm{CO}_{2}$ absorption rate at about 60 minutes. The absorption rate of the three solutions is in the following order: $20 \mathrm{wt} \%>15 \mathrm{wt} \%>10 \mathrm{wt} \%$.

Fig. 4 shows the variations in the absorption rate with increases in the $\mathrm{CO}_{2}$ absorption in the solutions. When the $\mathrm{CO}_{2}$ content in the solution was less than $0.6 \mathrm{~mol}$, the AEE solution with a low mass fraction $(10 \mathrm{wt} \%)$ had a higher $\mathrm{CO}_{2}$ absorption rate $(10 \mathrm{wt} \%>15 \mathrm{wt} \%>20 \mathrm{wt} \%)$, with the same $\mathrm{CO}_{2}$ absorption amount. Throughout the entire absorption reaction process, the change in the solution absorption rate was more dramatic in the early stage of the reaction; the changes in the $\mathrm{CO}_{2}$ absorption rate were fewer in the middle stage, and the changes in the absorption rate were more dramatic in the later stage. There are two main reasons for this. On the one hand, the higher mass fraction of AEE will lead to an increase in the viscosity of the solution and in turn affect the mass transfer effect; on the other hand, as the absorption reaction continues, the water content increases, which is conducive to the formation of carbamate and an increase in the absorption rate of $\mathrm{CO}_{2}$ in the solution. It can be concluded that proper reduction of the mass fraction of the AEE solution is beneficial to improve the absorption of $\mathrm{CO}_{2}$.

Fig. 5 shows that the change in the $\mathrm{pH}$ of the solution with time was almost linear 20 minutes prior to the start of the reaction. This is related to the rapid reaction of $\mathrm{OH}^{-}$and $\mathrm{CO}_{2}$

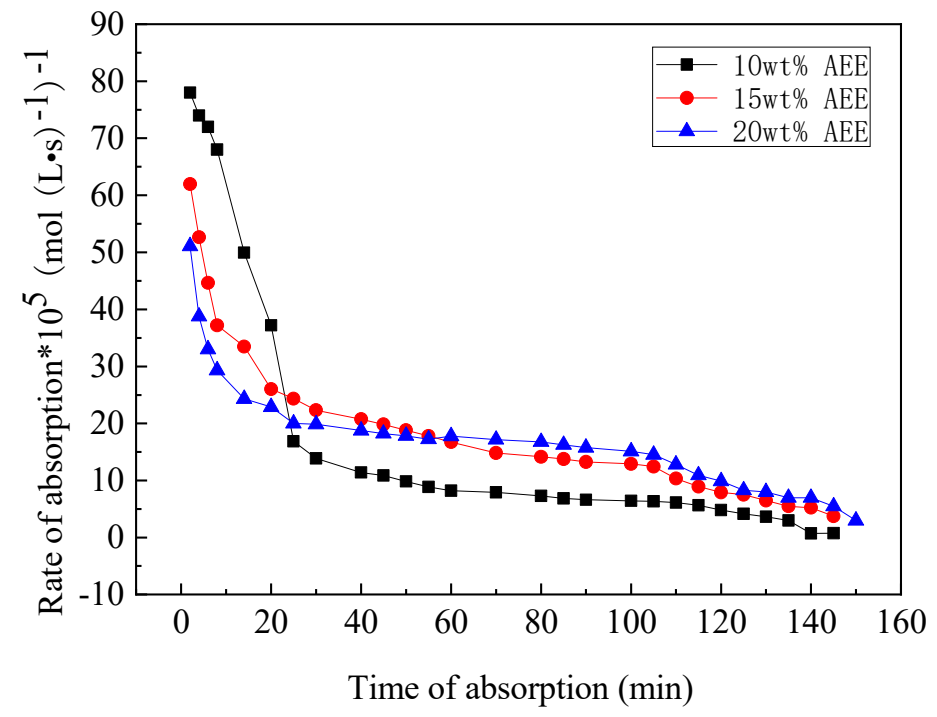

Fig. 3. Effects of experimental time on AEE solution absorption rate at different concentrations.

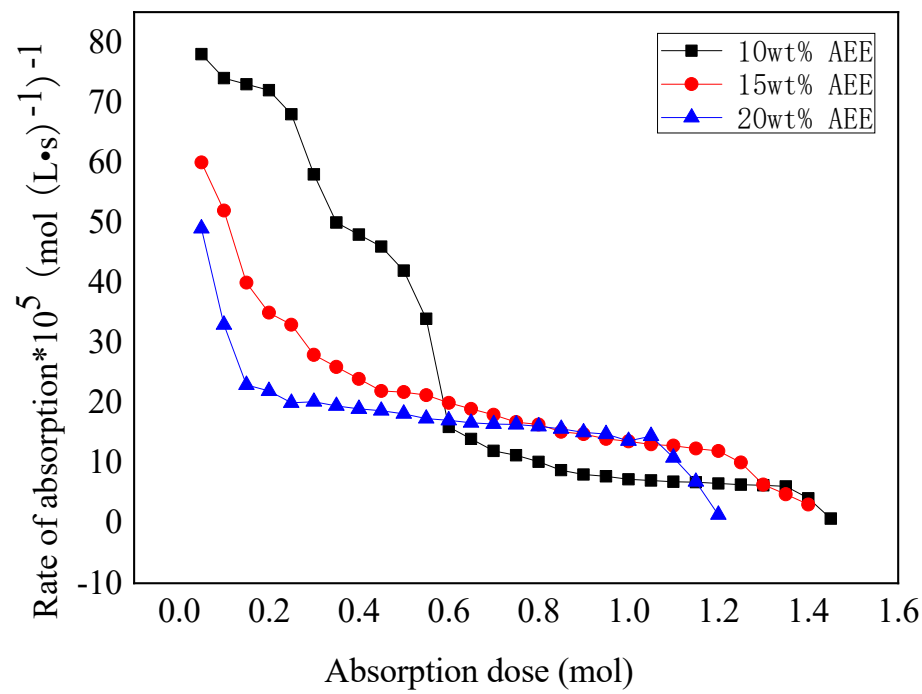

Fig. 4. Effects of amount of absorption on the absorption rate of AEE solutions at different concentrations. 


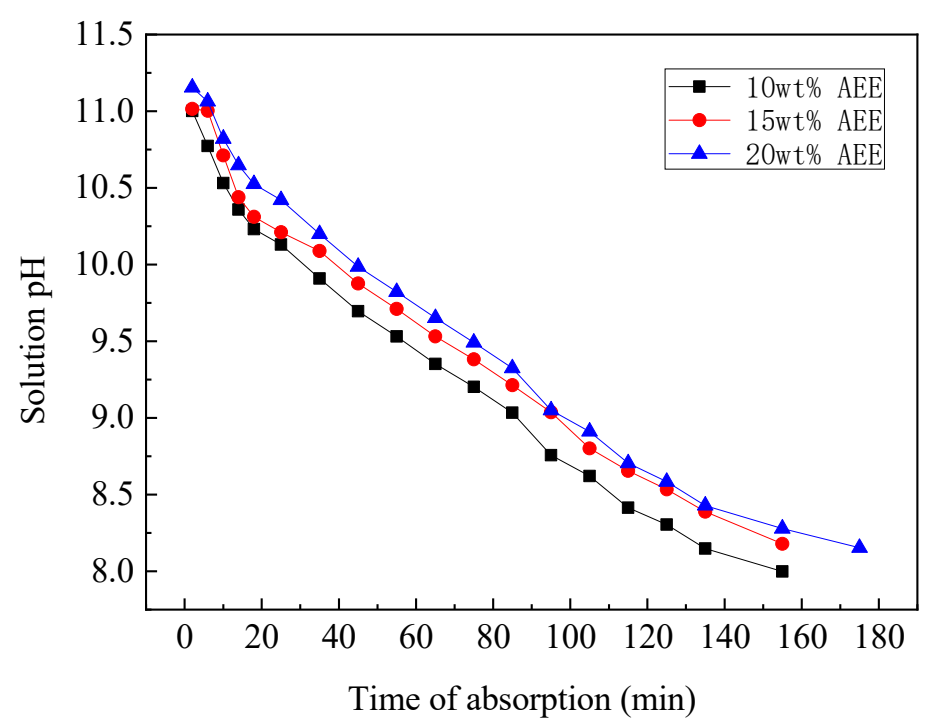

Fig. 5. Effects of experimental time on the AEE solution $\mathrm{pH}$ at different AEE solution concentrations.

in the AEE solution and the rapid decrease in the solution concentration. As the reaction time proceeded, the main constituent of the $\mathrm{CO}_{2}$ absorbent was converted to AEE molecules. $\mathrm{OH}^{-}$can inhibit the ionic absorption of AEE. As the alkalinity of the solution decreases, the AEE molecule will gradually release, which buffers the decrease in acidity and alkalinity of the solution and slows down the decrease in the $\mathrm{pH}$. This characteristic can be used as an index by which to judge the extent of $\mathrm{CO}_{2}$ absorption. When the $\mathrm{pH}$ of the solution drops to approximately 8.2, the absorption solution basically reaches saturation and cannot continue to absorb $\mathrm{CO}_{2}$ well, allowing it to be regenerated.

\section{Experiment on $\mathrm{CO}_{2}$ Desorption Using AEE Solutions}

Desorption is a mass transfer operation that releases solutes from solution under specific operating conditions. It is an inverse absorption process. Generally speaking, the desorption reaction can obtain the absorption solution of the re-reaction and make it possible to obtain a relatively pure gas solute. In order to objectively evaluate the $\mathrm{CO}_{2}$ desorption performance of AEE solutions with different concentrations at room temperature and pressure, AEE solutions with mass fractions of $10 \mathrm{wt} \%, 15 \mathrm{wt} \%$, and $20 \mathrm{wt} \%$ were selected to conduct the $\mathrm{CO}_{2}$ absorption experiments. The results are shown in Figs. 6 to 10.

Figs. 6 and 7 show the effects of experimental time and temperature on the desorption rate of AEE solutions at different concentrations. In the first 30 minutes, the temperature of the solution continued to rise, during which the desorption rates of the different concentrations of AEE solution reached their maximum levels. From Fig. 7, it can be seen that the desorption rate of the various AEE solutions reached the maximum at about $85^{\circ} \mathrm{C}$, where the desorption rate of the AEE solutions was relatively high in the range of $85^{\circ} \mathrm{C}$ to $95^{\circ} \mathrm{C}$. The range of temperatures was relatively wide. With a rapid increase in the solution temperature, the desorption rate changed dramatically and rapidly reached its maximum. Then, with continuous increases in temperature, the desorption and escape of $\mathrm{CO}_{2}$ in the solution gradually decreased; the desorption power decreased; the desorption rate decreased, and the reaction was weak. From Fig. 6, within the first 100 minutes of desorption, the desorption rates were $20 \mathrm{wt} \%$ $>15 \mathrm{wt} \%>10 \mathrm{wt} \%$. To summarize, after 30 minutes of heating, the desorption rate of the solution was higher when the temperature rose to $85^{\circ} \mathrm{C}$. The experimental results can be used as a guide for industrial selection of the appropriate desorption temperature.

The desorption energy consumption and time variations for the AEE solutions with different mass fractions are shown in Fig. 8. The mass fraction of the AEE solution affected the desorption energy consumption and determines the initial energy consumption, which was $20 \mathrm{wt} \%>15 \mathrm{wt} \%>10 \mathrm{wt} \%$. The energy consumption of the solution decreased rapidly in the initial 25 minutes and achieved a lower energy consumption level, indicating the good desorption performance of the solution. The three curves show a more consistent trend in the form of a "U" shape. This is because during the first 25 minutes of desorption, the temperature of the solution continued to rise. With this rapid increase in the solution temperature, the desorption energy consumption decreased rapidly. After 30 minutes, the optimal desorption condition was achieved, and the desorption energy consumption was in a relatively stable period, maintaining a low value and demonstrating the best desorption effect. Among the different solutions, the $20 \mathrm{wt} \%$ AEE maintained the minimum desorption energy consumption for the least time, approximately 70 minutes, while the $10 \mathrm{wt} \%$ and $15 \mathrm{wt} \%$ AEE solutions lasted about 100 minutes and has a large operating space.

The relationship between the energy consumption due to AEE solution desorption and the content of $\mathrm{CO}_{2}$ in the AEE solutions is shown in Fig. 9. The three curves are similar, showing a "U" shape. When the content of $\mathrm{CO}_{2}$ in the solution was high, the solution exhibited higher desorption energy consumption. At this time, the main factor determining the energy consumption was the solution temperature. When the temperature of the solution reached the appropriate working 


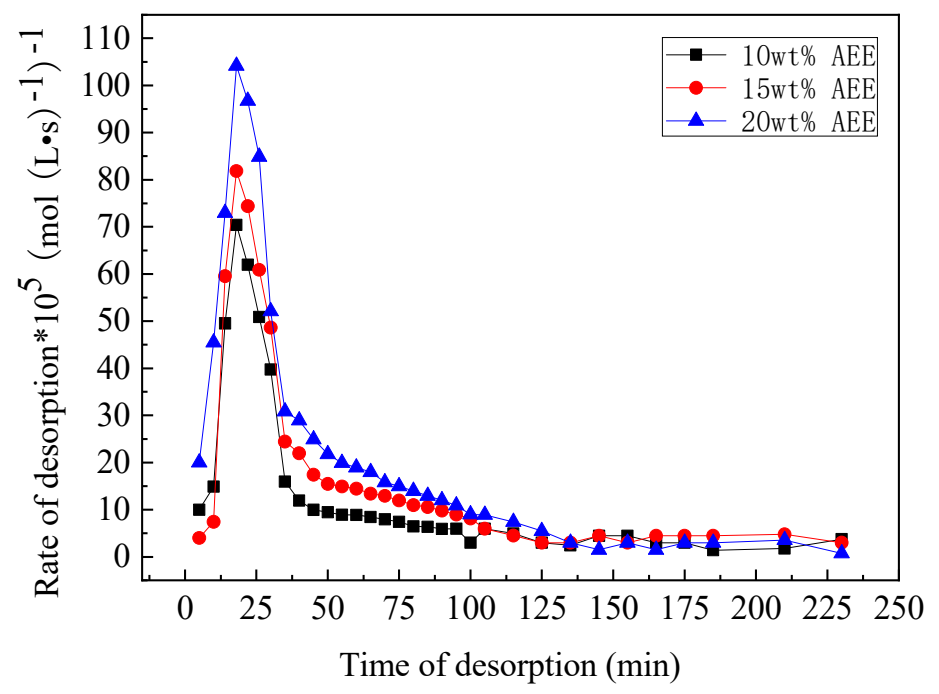

Fig. 6. Effect of desorption time on the desorption rate of AEE solutions at different concentrations.

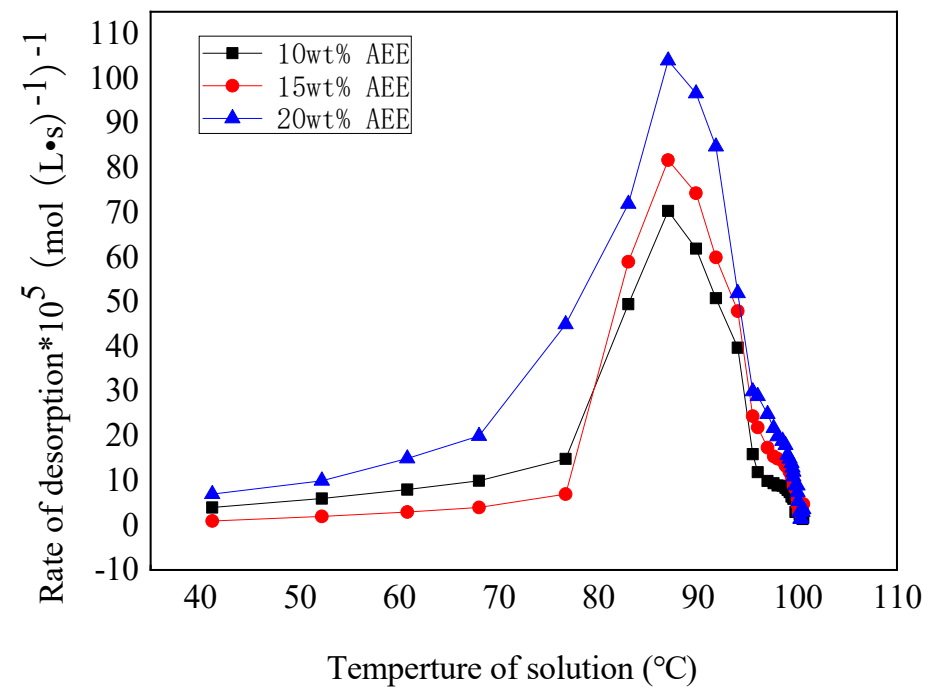

Fig. 7. Effect of the solution temperature on the desorption rate of AEE solutions at different concentrations.

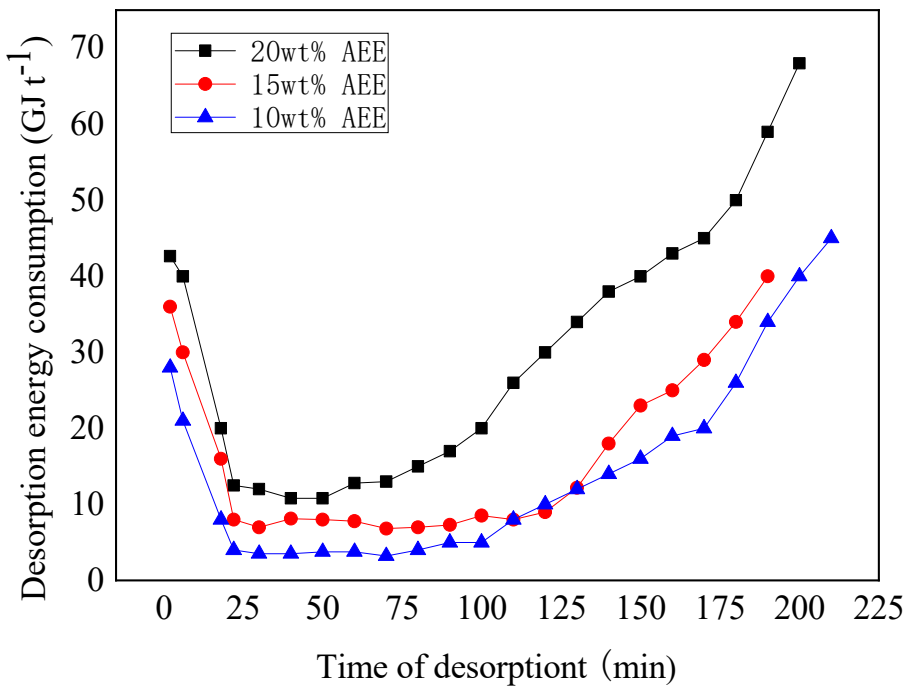

Fig. 8. Effects of experimental time on the desorption energy consumption of AEE solutions at different concentrations. 


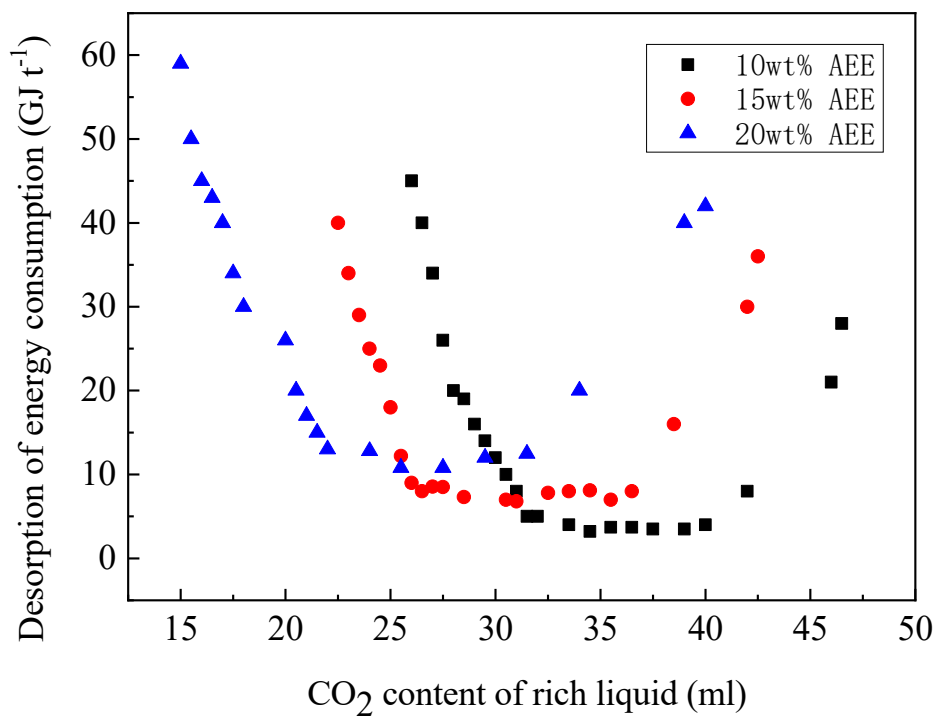

Fig. 9. Effect of $\mathrm{CO}_{2}$ content on desorption energy consumption of AEE solutions at different concentrations.

conditions, the factors determining the energy consumption of the solution changed to the content of $\mathrm{CO}_{2}$ in the solution. At the beginning of the desorption reaction, the solution had a higher concentration of $\mathrm{CO}_{2}$, but at this time, the solution temperature was lower, so the desorption energy consumption was higher. As the reaction proceeded, the total $\mathrm{CO}_{2}$ content in the solution decreased, and the energy consumption per unit of $\mathrm{CO}_{2}$ desorption rapidly increased. Therefore, the content of $\mathrm{CO}_{2}$ in the alkanol-amine solution also reflects the energy consumption level of desorption at a given time. Choosing suitable conditions under which to start and end the desorption process is therefore of great significance in terms of reducing energy consumption in industrial applications.

The curves for the degree of regeneration of the AEE solution with time are shown in Fig. 10. Within 20 minutes prior to the desorption reaction, the temperature of the solution was lower; the desorption reaction was not fully carried out, and the regeneration degree was lower. However, a rapid growth trend can be observed, which is consistent with the maximum desorption rate in the first 20 minutes. With the passage of time, the temperature of the solution increased; the content of $\mathrm{CO}_{2}$ in the solution decreased; the desorption rate decreased, and the trend in the increase of the degree of regeneration slowed. In the middle stage, the degree of regeneration was in the following order: $20 \mathrm{wt} \%>15 \mathrm{wt} \%$ $>10 \mathrm{wt} \%$. Among the proposed solutions, the $20 \mathrm{wt} \%$ AEE solution had strong regeneration ability. In the later stage of desorption, however, the three were basically equal.

\section{Experimental Analysis of Three Cycles of Absorption and Desorption of $\mathrm{CO}_{2}$ for the $10 \mathrm{wt} \% \mathrm{AEE}$ Solution}

The $10 \mathrm{wt} \%$ AEE solution was selected as the research object. The absorption and desorption properties of the AEE solution for three cycles were analyzed experimentally, and the changes in the AEE solution were revealed by comparing the three cycles. The results are shown in Figs. 11 to 14.

Fig. 11 shows that the absorption rate curves for the $10 \mathrm{wt} \%$ AEE for three cycles differs significantly in the first
30 minutes. The fresh AEE solution had a relatively high absorption rate. After 30 minutes of absorption, the curves basically merge, showing consistency. This indicates that the main factor affecting the absorption rate of the solution is the $\mathrm{CO}_{2}$ loading.

From Fig. 12, it can be concluded that the $\mathrm{pH}$ values for the AEE three-cycle absorption were basically equal, where the activity of the solution decayed layer by layer after each absorption and desorption process, which suggests good conditions for industrial application.

From Fig. 13, it can be concluded that the trend in the desorption rate for all three cycles was basically the same. 100 minutes before the reaction, the difference in the desorption rate for the three cycles was obvious, followed by the third cycle being greater than the second cycle, and the second cycle being greater than the first cycle. After 100 minutes, these differences were insignificant.

Fig. 14 shows that the desorption energy consumption of the AEE solution was generally at a relatively low level, and the time of the low energy consumption was longer, especially from 25 minutes to 100 minutes, where it was only $5 \mathrm{GJ} / \mathrm{t}$, while from 100 minutes to 175 minutes, it was 10 to $20 \mathrm{GJ} / \mathrm{t}$, and the desorption energy consumption was much lower than that of the first three solutions. The low energy consumption process lasted for a substantial period of time, which easily gave full play to its advantages. After three cycles of experiments, the curve change law was more consistent, and the desorption performance was relatively stable, showing that the solution can be reused many times. The desorption rate and energy consumption of the AEE solution were very good, which infers great industrial application value.

\section{CONCLUSIONS}

In this work, based on $\mathrm{CO}_{2}$ capture in the flue gas from coal-fired power plants, a laboratory experiment platform was built based on the on-site capture process. In the experiment, the chemical absorption method was adopted to 


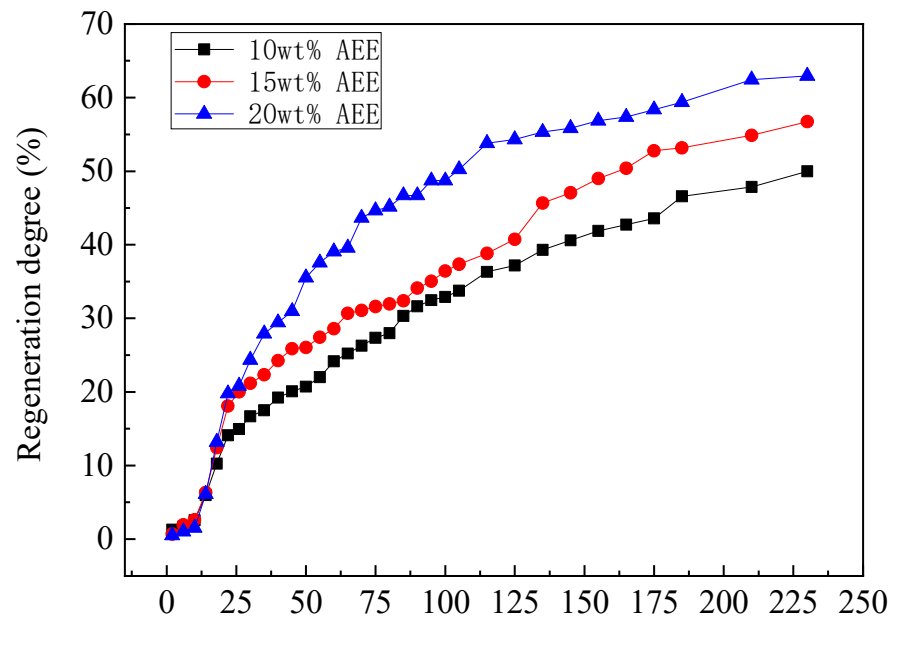

Time of desorption (min)

Fig. 10. Effect of experimental time on the degree of regeneration for AEE solutions at different concentrations.

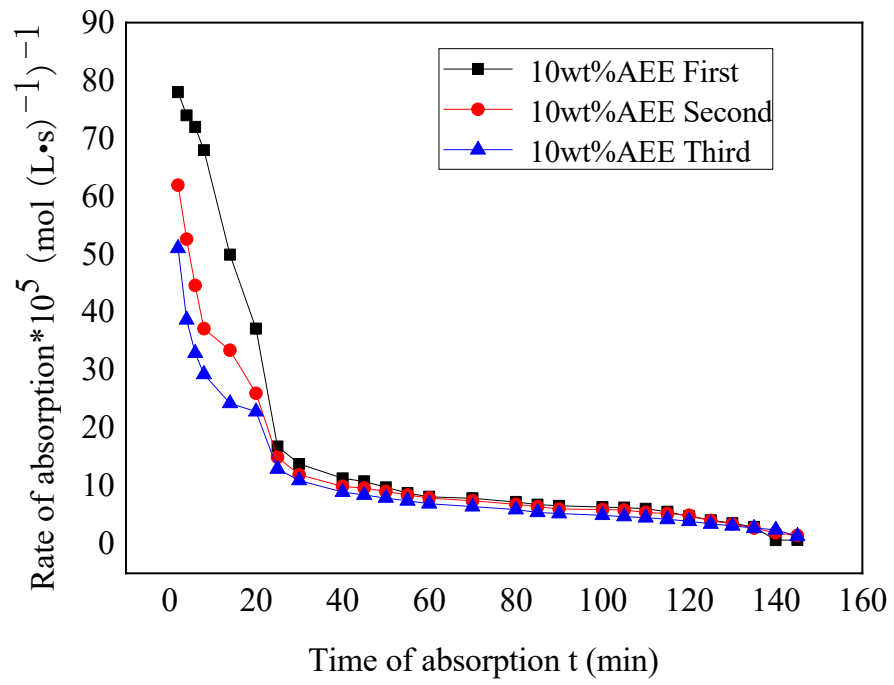

Fig. 11. Effect of experimental time on the absorption rate for three cycles rate of the $10 \mathrm{wt} \%$ AEE solution.

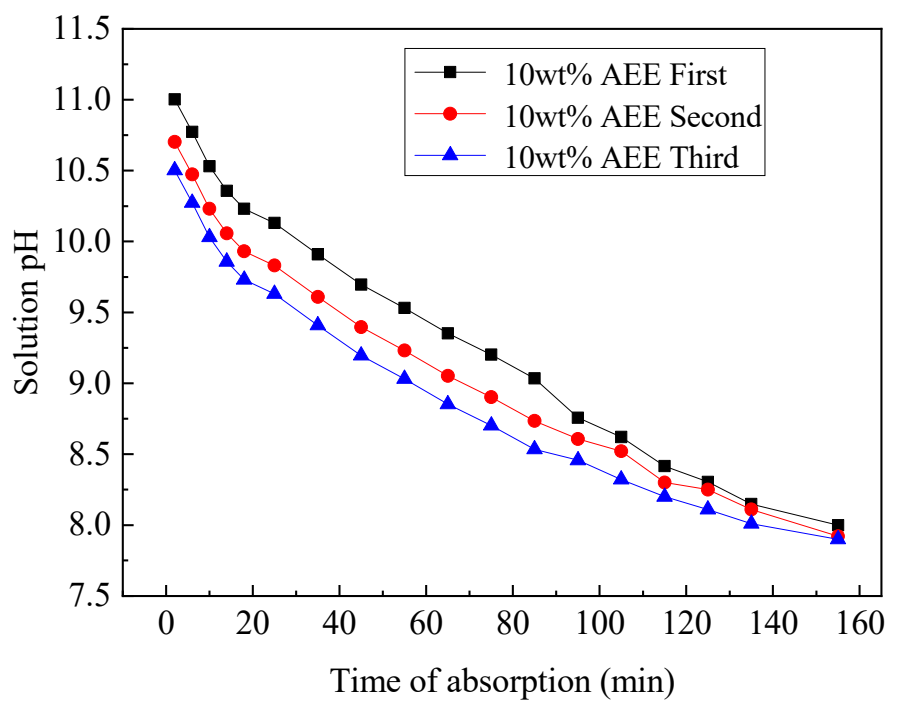

Fig. 12. Effect of experimental time on the solution $\mathrm{pH}$ after three cycles for the $10 \mathrm{wt} \% \mathrm{AEE}$. 


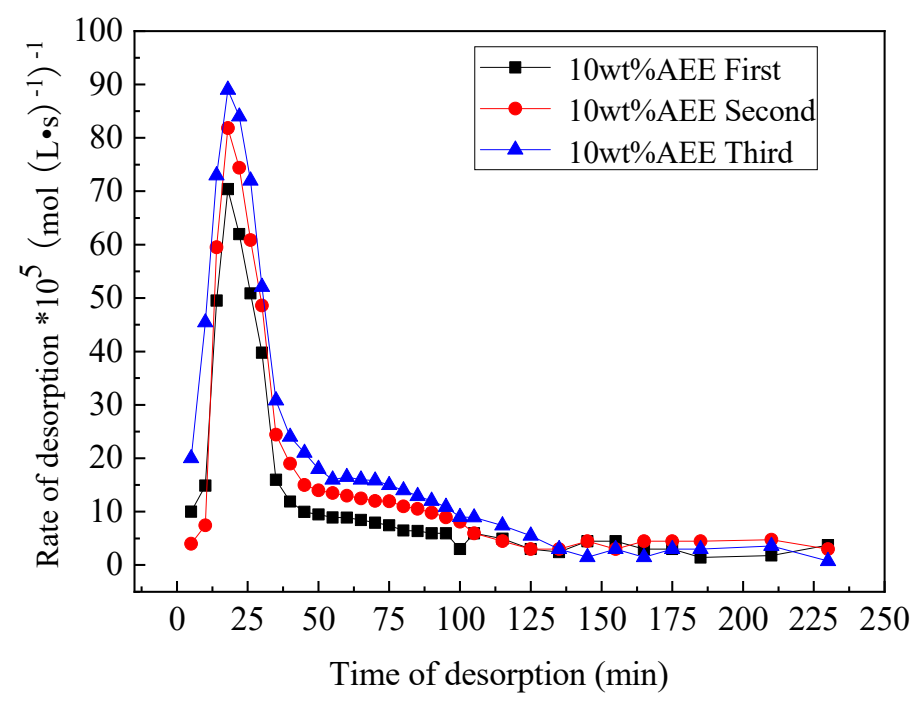

Fig. 13. Effects of experimental time on desorption rate for three cycles of the $10 \mathrm{wt} \% \mathrm{AEE}$ solution.

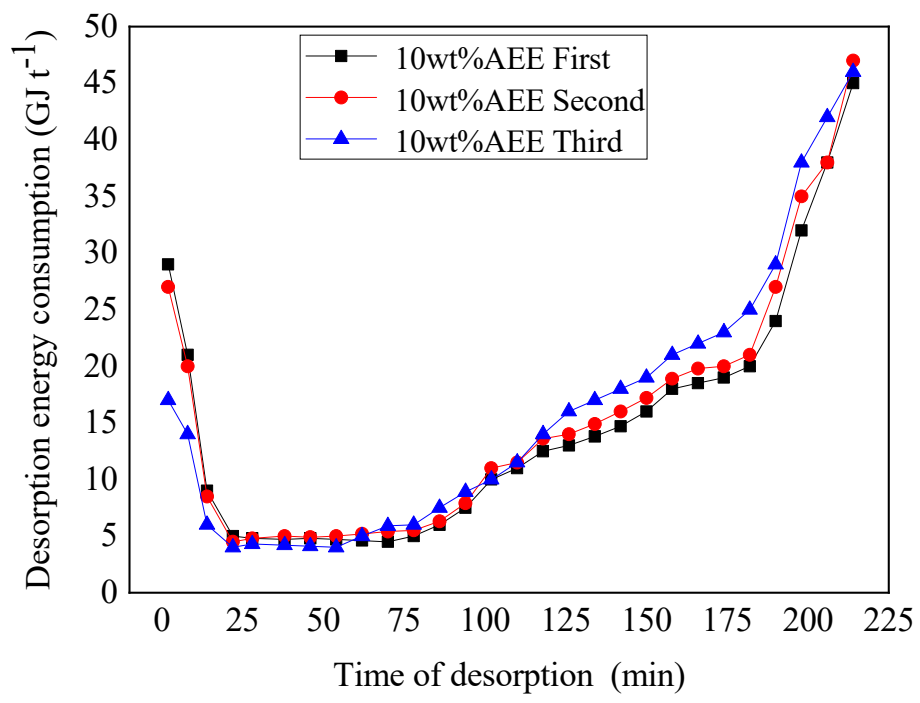

Fig. 14. Effect of experimental time on three cycles of desorption energy consumption for the $10 \mathrm{wt} \%$ AEE solution.

simulate the flue gas from power plants, and the chemical absorbent used was the alcohol amine solution, AEE, as commonly used in industrial practice. The main findings for the AEE amine solution are as follows:

(1) As the absorption experiment proceeds, the solution with a mass fraction of $20 \mathrm{wt} \%$ at about $60 \mathrm{~min}$ has a higher $\mathrm{CO}_{2}$ absorption rate, and the absorption rate decreases with increases in the amount of absorption, so proper reduction of the mass fraction of the AEE solution is beneficial to improving the absorption of $\mathrm{CO}_{2}$ using the AEE solution.

(2) As the desorption experiment proceeds, within the first $100 \mathrm{~min}$ of desorption, the desorption rates were $20 \mathrm{wt} \%$ $>15 \mathrm{wt} \%>10 \mathrm{wt} \%$. At $25 \mathrm{~min}$, the desorption rates of different concentrations of the AEE solution reach their maximum values when the temperature rises to $85^{\circ} \mathrm{C}$, while the desorption energy consumption is low. The desorption low energy consumption process lasts for a significant period of time.

(3) Through three cycles of absorption and desorption of the AEE solution, the AEE solution initially has a relatively high absorption rate, but a relatively high desorption rate occurs in the third use. In terms of energy consumption, the desorption energy consumption of the AEE solution is generally at a relatively low level and will not cause greater desorption energy consumption due to recycling, especially in the range of $25 \mathrm{~min}-100 \mathrm{~min}$, where it is only $5 \mathrm{GJ} / \mathrm{t}$.

In this paper, the determination of the performance of the AEE solution provides a better choice than other alternatives for the absorption of $\mathrm{CO}_{2}$ using a chemical method and an alcohol-amine solvent. The experiment proves that an AEE solution has advantages including low energy consumption and desorption, which is of great significance for industrial application. However, in the experiment, one limitation was that the heating mode was circular heat conduction oil 
heating, and its desorption was therefore not thorough enough, which is different from what would occur in actual industrial production. Therefore, these challenges will be discussed and optimized in future works.

\section{ACKNOWLEDGMENTS}

This work was supported by Department of Science \& Technology of Shandong Province (No. ZR2018LB025).

\section{REFERENCES}

Afkhamipour, M., Mofarahi, M., Rezaei, A., Mahmoodi, R. and Lee, C.H. (2019). Experimental and theoretical investigation of equilibrium absorption performance of $\mathrm{CO}_{2}$ using a mixed 1-dimethylamino-2-propanol (1DMA2P) and monoethanolamine (MEA) solution. Fuel 256: 115877.

Anderson, S. and Newell, R. (2004). Prospects for Carbon Capture and Storage Technologies. Annu. Rev. Environ. Resour. 29: 109-142.

Azhgan M., Farsi M. and Eslamloueyan R. (2016). Solubility of carbon dioxide in aqueous solution of 1,5diamino-2-methylpentane: Absorption and desorption property. Int. J. Greenhouse Gas Control 51: 409-414.

Bamdad, H., Hawboldt, K. and MacQuarrie, S. (2018). A review on common adsorbents for acid gases removal: Focus on biochar. Renewable Sustainable Energy Rev. 81: 1705-1720.

Beidari M., Lin, S.J. and Lewis, C. (2017). Decomposition analysis of $\mathrm{CO}_{2}$ emissions from coal - Sourced electricity production in South Africa. Aerosol Air Qual. Res. 17: 1043-1051.

Bonenfant, D., Mimeault, M. and Hausler, R. (2005). Comparative analysis of carbon dioxide absorption and recuperation capacities in aqueous 2-(2-aminoethyamina) ethanol (AEE) and blends of aqueous (AEE) and nmethldiethanolamine solutions. Ind. Eng. Chem. Res. 44: 3720-3725.

Butterfield, D.M. and Quincey, P. (2017). An investigation into the effects of off-shore shipping emissions on coastal black carbon concentrations. Aerosol Air Qual. Res. 17: 218-229.

Caplow, M. (1968). Kinetics of carbamate formation and breakdown. J. Am. Chem. Soc. 90: 6795-6803.

Chatterjee, A., Roy, A., Chakraborty, S., Karipot, A.K., Sarkar, C., Singh, S., Ghosh, S.K., Mitra, A. and Raha, S. (2018). Biosphere atmosphere exchange of $\mathrm{CO}_{2}, \mathrm{H}_{2} \mathrm{O}$ vapour and energy during spring over a high altitude Himalayan forest in Eastern India. Aerosol Air Qual. Res. 18: 2704-2719.

Danckwerts, P.V. (1979). The reaction of $\mathrm{CO}_{2}$ with ethanolamines. Chem. Eng. Sci. 34: 443-446.

Gai, Q.Y., Zhang, Y.C., Zhou, J.X., Song, W. and Wang, C.X. (2008). Study on absorption and desorption of $\mathrm{CO}_{2}$ from alcohol amine solution. Environ. Pollut. Prev. 30: 62-65.

Ghasemifard, H., Yuan, Y., Luepke, M., Schunk, C., Chen, J., Ries, H., Leuchner, M. and Menze, A. (2019).
Atmospheric $\mathrm{CO}_{2}$ and $\delta^{13} \mathrm{C}$ measurements from 2012 to 2014 at the environmental research station schneefernerhaus, Germany: Technical corrections, temporal variations and trajectory clustering. Aerosol Air Qual. Res. 19: 657-670.

Goff, G.S and Rochelle, G.T. (2004). Monoethanolamine degradation: $\mathrm{O}_{2}$ mass transfer effects under $\mathrm{CO}_{2}$ capture conditions. Ind. Eng. Chem. Res. 43: 6400-6408.

Hikita H., Asai S., Katsu Y, and Lkuno, S. (1979). Absorption of carbon dioxide into aqueous monoethanolamine solutions. AIChE J. 25: 793-800.

International Energy Agency (IEA) (2017). $\mathrm{CO}_{2}$ Emissions from Fuel Combustion Highlights (2017 edition). International Energy Agency, France.

Issacs, E.E., Otto, F.D. and Mather, A.E. (1980). Solubility of mixtures of $\mathrm{H}_{2} \mathrm{~S}$ and $\mathrm{CO}_{2}$ in a monoethanolamine solution at low partial pressures. J. Chem. Eng. 25: 118120.

Jain, C.D., Gadhavi, H.S., Wankhede, T., Kallelapu, K., Sudhesh, S., Das, L.N., Pai, R.U. and Jayaraman, A. (2018). Spectral properties of black carbon produced during biomass burning. Aerosol Air Qual. Res. 18: 671679.

Jamal, A., Meisen, A. and Lim, C.J. (2006). Kinetics of carbon dioxide absorption and desorption in aqueous alkanolamine solutions using a novel hemispherical contactor-I. Experimental apparatus and mathematical modeling. Chem. Eng. Sci. 61: 6590-6603.

Kato, Y., Murai, S., Muraok, D., Muramatsu, T. and Saito, S. (2013). Evaluation of carbon dioxide absorption by amine based absorbent. Energy Procedia 37: 325-330.

Lee, C.M. and Rosalez, E.R. (2017). Economic growth, carbon abatement technology and decoupling strategy The case of Taiwan. Aerosol Air Qual. Res. 17: 15491557.

Li, L., Zhao, N., Wei, W. and Sun, Y. (2013). A review of research progress on $\mathrm{CO}_{2}$ capture storage, and utilization in Chinese Academy of Sciences. Fuel 108: 112-130.

Li, P., Pan, S.Y., Pei, S., Lin, Y.J. and Chiang, P.C. (2016). Challenges and perspectives on carbon fixation and utilization technologies: An overview. Aerosol Air Qual. 16: 1327-1344.

Liang, Z., Rongwong, W., Liu, Fu, K., Gao, H. and Cao, F. (2015). Recent progress and new developments in postcombustion carbon-capture technology with amine based solvents. Int. J. Greenhouse Gas Control 40: 26-54.

Lin, P.H. and Wong, D.S.H. (2014). Carbon doxide capture and regeneration with amine/alcohol/water blends. Int. J. Greenhouse Gas Control 26: 69-75.

Liu, X. and Bae, J. (2018). Urbanization and industrialization impact of $\mathrm{CO}_{2}$ emissions in China. J. Cleaner Prod. 172: $178-186$.

Lund, H. and Mathiesen, B.V. (2012). The role of carbon capture and storage in a future sustainable energy system. Energy 44: 469-476.

Melnikov, S.M. and Stein, M. (2019). The effect of $\mathrm{CO}_{2}$ loading on alkanolamine absorbents in aqueous solutions. Phys. Chem. Chem. Phys. 21: 18386-18392.

Monda, M.K., Balsora, H.K. and Varshney, P. (2012). Progress and trends in $\mathrm{CO}_{2}$ capture/separation technologies: 
A review. Energy 46:431-41.

Pan, X., Clodic, D. and Toubassy, J. (2013). $\mathrm{CO}_{2}$ capture by antisublimation process and its technical economic analysis. Greenhouse Gases Sci. Technol. 3: 8-20.

Rochelle, G.T. (2009). Amine scrubbing for $\mathrm{CO}_{2}$ capture. Science 325: 1652-1654.

Shakerian, F., Kim, K.H. Szulejko, J.E. and Park J.W. (2015). A comparative review between amines and ammonia as sorptive media for post-combustion $\mathrm{CO}_{2}$ capture. Appl. Energy 148: 10-22.

Song, C.F., Kitamura, Y., Li, S.H. and Jiang, W.Z. (2012). Parametric analysis of a novel cryogenic $\mathrm{CO}_{2}$ capture system based on Stirling coolers. Environ. Sci. Technol. 46: $12735-12741$.

Su, C.M., Hsueh, H.T., Tseng, C.M., Ray, D.T., Shen, Y.H. and $\mathrm{Chu}, \mathrm{H}$. (2017). Effects of nutrient availability on the biomass production and $\mathrm{CO}_{2}$ fixation in a flat plate photobioreactor. Aerosol Air Qual. Res. 17: 1887-1897.

Szulczewski, M.L., MacMinn, C.W., Herzog, H.J. and Juanes, R. (2012). Lifetime of carbon capture and storage as a climate-change mitigation technology. Proc. Natl. Acad. Sci. U.S.A. 109: 5185-5189.

Wang, M., Wang, Z., Zhao, S., Wang, J. and Wang, S. (2017). Recent advances on mixed matrix membranes for $\mathrm{CO}_{2}$ separation. Chin. J. Chem. Eng. 25: 1581-1597.

Xie, W.J., Yu, M.Q. and Wang, R. (2017). $\mathrm{CO}_{2}$ capture behaviors of amine-modified resorcinol-based carbon aerogels adsorbents. Aerosol Air Qual. Res. 17: 2715-
2725.

Yancheshmeh, M.S., Radfarnia, H.R. and lliuta, M.C. (2016). High temperature $\mathrm{CO}_{2}$ sorbents and their application for hydrogen production by sorption enhanced steam reforming process. Chem. Eng. J. 283: 420-444.

Yang, W.B., Yuan, C.S., Chen, W.H., Yang, Y.H. and Hung, C.H. (2017). Diurnal variation of greenhouse gas emission from petrochemical wastewater treatment processes using in-situ continuous monitoring system and the associated effect on emission factor estimation. Aerosol Air Qual. Res. 17: 2608-2623.

Yu, C.H., Hung, C.H. and Tan, C.H. (2012). A review of $\mathrm{CO}_{2}$ capture by absorption and adsorption. Aerosol Air Qual. Res. 12: 745-769.

Zhang, X., Liu, H., Liang, Z., Idem, R., Tontiwachwuthikul, P., Al-Marri, M.J. and Benamor, A. (2018). Reducing energy consumption of $\mathrm{CO}_{2}$ desorption in $\mathrm{CO}_{2}$-loaded aqueous amine solution using $\mathrm{Al}_{2} \mathrm{O}_{3} / \mathrm{HZSM}-5$ bifunctional catalysts. Appl. Energy 229: 562-576.

Zhang, Y., Sunarso, J., Liu, S. and Wang, R. (2013). Current status and development of membranes for $\mathrm{CO}_{2} / \mathrm{CH}_{4}$ separation: A review. Int, J Greenh Gas Control. 12: 84107.

Received for review, November 4, 2019

Revised, November 15, 2019

Accepted, November 16, 2019 